\title{
Recent Shifts in Racial/Ethnic Disparities in COVID-19 Mortality in the Vaccination Period in California
}

KEY WORDS: COVID-19; vaccination; proportionate mortality; racial disparities; health equity.

J Gen Intern Med 37(7):1818-20

DOI: $10.1007 / \mathrm{s} 11606-021-07380-6$

(C) The Author(s) under exclusive licence to Society of General Internal Medicine 2022

\section{INTRODUCTION}

The first year of the COVID-19 pandemic was marked by extraordinary racial/ethnic disparities in mortality ${ }^{1}$. COVID19 mortality in California began to decline after January 2021. It is unknown if these rapid declines in mortality mitigated or exacerbated racial/ethnic disparities in COVID-19 mortality.

There is limited evidence on how racial/ethnic disparities in COVID-19 mortality shifted in the vaccination period. Welldocumented inequities in vaccine uptake ${ }^{2}$ may have exacerbated racial/ethnic disparities, but the combination of vaccine hesitancy in some predominantly white communities ${ }^{3}$ and lower immunity from past infection may have closed gaps between whites and other groups.

We evaluated how racial/ethnic disparities in COVID-19 mortality and proportionate mortality evolved with availability of the vaccine in California and whether these changes differed by age group. We used weekly proportionate mortality - defined as deaths in a specified population as a proportion of all deaths that week - as a relative measure of disparities.

\section{METHODS}

Analyses used California death certificates from 3/1/2020 to $7 / 31 / 2021$ and population estimates from the 2020 American Community Survey. We evaluated COVID-19 mortality for the four largest racial/ethnic groups in California: Latinos of any race, non-Latino white, Black, and Asian individuals. For each group, we estimated (1)age-standardized and agestratified mortality rates and (2) the weekly proportion of deaths in each group overall and within three age strata (hereafter, race-age subgroups). We fit joinpoint regression to

Alicia R. Riley and Mathew V. Kiang contributed equally to this work. Alicia R. Riley and Mathew V. Kiang are joint-first authors.

Received December 6, 2021

Accepted December 17, 2021

Published online February 2, 2022 quantify trends in the COVID-19 mortality rate during the vaccination period (starting 1/13/2021). We fit weighted quartic polynomial models to estimate trends in the proportion of COVID-19 deaths for each race-age subgroup.

\section{RESULTS}

We estimated large rates of decline in COVID-19 mortality for all race-age subgroups in the vaccination period, ranging from $-23 \%$ (95\% CI: $-27,-19)$ weekly percent change (WPC) in Black individuals to $-25 \%(-27,-24)$ WPC in Latinos, followed by slower decreases in mortality in March for all race-age subgroups (Fig. 1). These declines reflect large reductions in the age-standardized mortality rate ranging from a 15 -fold reduction for whites to 48 -fold reduction for Latinos from mid-January to late July.

Among adults aged 40 and older, proportionate mortality increased for Latinos during the first weeks of the pandemic and decreased for all other racial/ethnic groups (Fig. 2), producing an equity gap that persisted until March. Beginning in March, the proportion of COVID-19 deaths occurring among Latinos aged 40 and older declined and the proportion of COVID-19 deaths occurring among whites of all ages increased. In the vaccination period, a growing fraction of decedents aged 40-64 were Black individuals (widening an equity gap). Smoothed trends indicate that Latinos comprised approximately $66 \%$ of COVID-19 decedents aged 40-64 in mid-March but only $30 \%$ of such decedents in late July. The smoothed trend for percent of white decedents aged 40-64 increased from 17 to $36 \%$ from March to July, and the percent Black increased from 6 to $21 \%$.

\section{DISCUSSION}

Age-standardized COVID-19 mortality rates have declined for all subgroups and racial/ethnic disparities have also shifted. The vastly disproportionate share of COVID-19 death that fell on Latino Californians for the first year of the pandemic progressively lessened in the vaccination period. Meanwhile, the share of COVID-19 deaths occurring in white subgroups steadily increased. These dynamics in the vaccination period appear to have closed equity gaps in COVID-19 proportionate mortality for Latinos and whites by July 2021. But mortality disadvantages persisted or grew for Black Californians, highlighting the need for attention to unchanged structural 

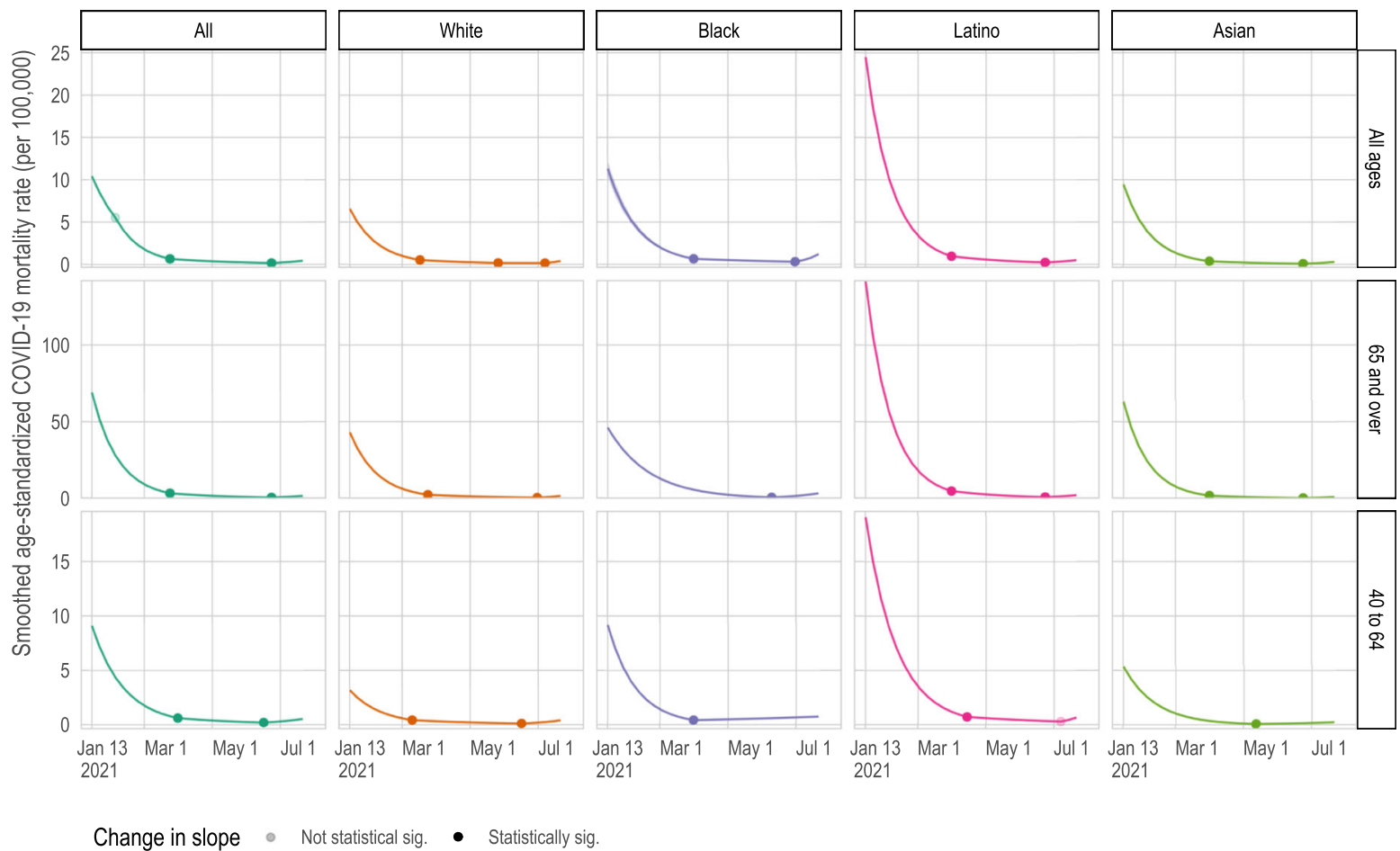

Change in slope • Not statistical sig. • Statistically sig

Figure 1 Age-standardized COVID-19 mortality rate trends by race/ethnicity and age. Note: smoothed trends estimated using joinpoint regression.

We fit weekly age-standardized and age-stratifiedCOVID-19-related mortality rates, by race/ethnicity, using joinpoint regression. Joinpoint regression detects natural changes (i.e., "joins") in linear trends that best fit observed data. Here, each dark filled circle represents a joinpoint that has a statistically significant change in slope while light circles represent detected changes in the slope that were not statistically significant.

Numeric results and model fit statistics are available in the code repository. Source: CA death certificates and 2020 American Community Survey.

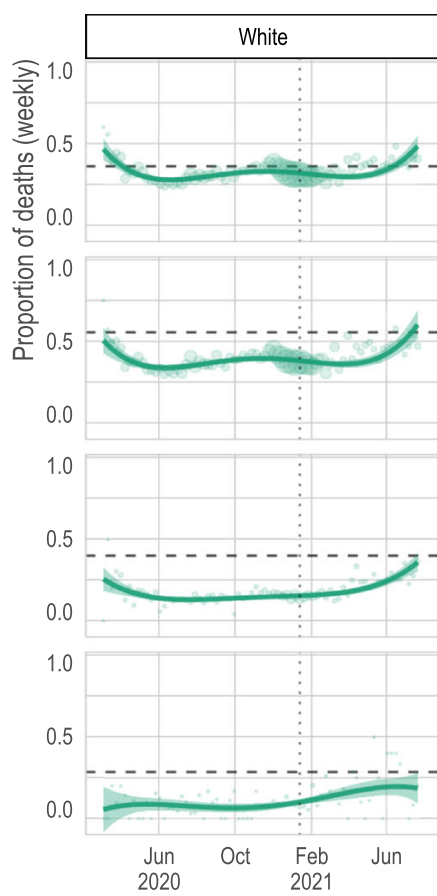

Number of COVID-19 deaths
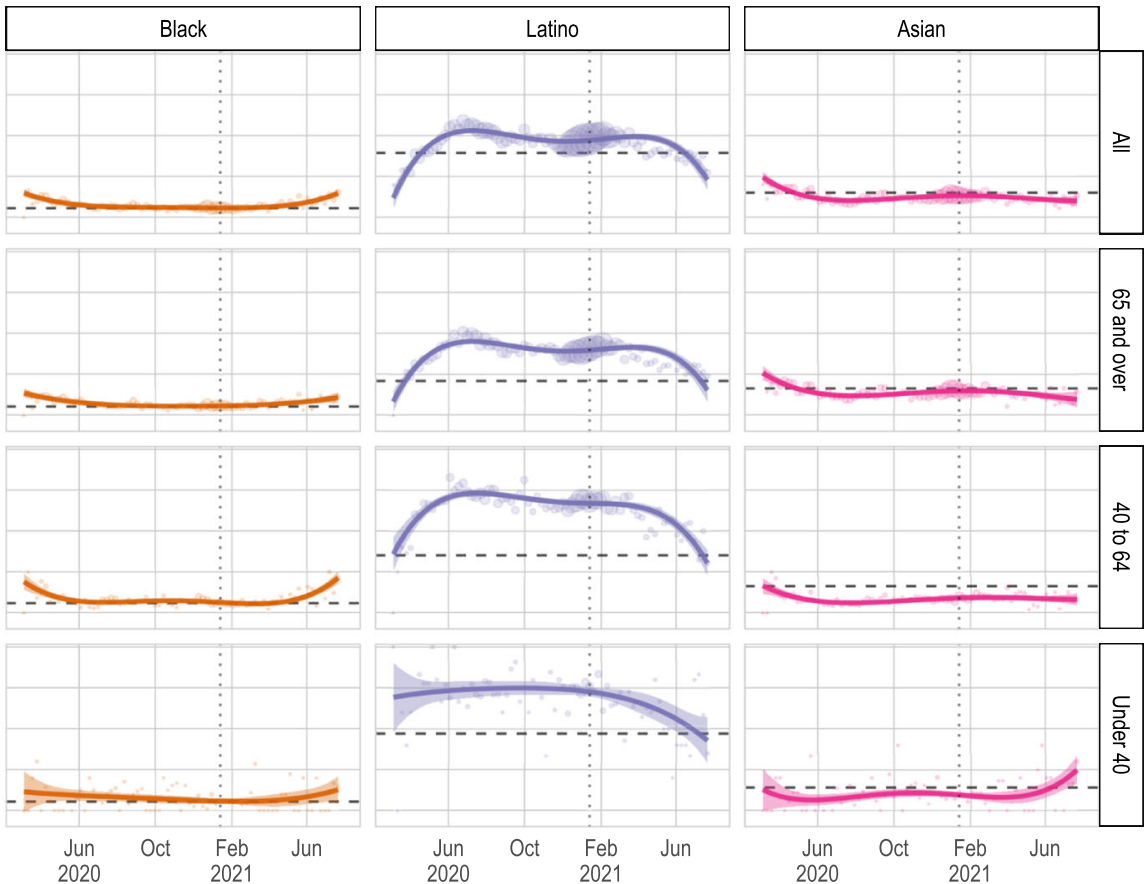

$<10$

100

$500 \bigcirc 1000$

1500

Figure 2 Proportionate COVID-19 mortality during the pandemic by race/ethnicity and age. Note: Smoothed trends estimated using polynomial regression. We fit weighted quartic polynomial regression to the proportion of weekly deaths for each race-age subgroup relative to the total number of weekly deaths, where the total number of weekly deaths were also the weights. Vertical dotted line indicates the date of vaccine authorization for general population aged 65 and older, January 13, 2021. Horizontal dashed line indicates proportion of the total California population comprised by each subgroup. Source: CA death certificates and 2020 American Community Survey. 
inequities. Asian Californians have comprised a lower fraction of COVID-19 deaths than their representation in the population since May 2021.

The trends reflect both vaccination and other forces. Pace of vaccination varied by group with Asian Californians reaching $50 \%$ fully vaccinated on $5 / 3 / 2021$, while white, Black, and Latino proportions vaccinated were $41 \%, 26 \%$, and $24 \%$, respectively ${ }^{4}$. Elevated COVID-19 infection rates in Latino communities may have meaningfully increased natural immunity ${ }^{5}$. Vaccine outreach in California may have helped narrow equity gaps for Latinos, reflecting tremendous resources invested by community organizations ${ }^{6}$, with help from state funding. Further investment and policy efforts to increase vaccination take-up across racial/ethnic groups and reduce structural inequities in exposure are needed.

Mortality disparities in COVID-19 are not inevitable. Racial/ethnic disparities in COVID-19 mortality can transform quickly with changes in structural factors, policy, behaviors, and patterns in exposure.

Acknowledgements: We thank the California Department of Public Health for providing data necessary for these analyses.

Alicia R. Riley, PhD, $M P H^{1}$

Mathew V. Kiang, $S c D^{2}$

Yea-Hung Chen, $\mathrm{PhD}^{3}$

Kirsten Bibbins-Domingo, PhD, MD, $M A S^{3}$

M. Maria Glymour, $\mathrm{ScD} \mathrm{D}^{3}$

${ }^{1}$ Department of Sociology, University of California, Santa Cruz,

Santa Cruz, California, USA

${ }^{2}$ Department of Epidemiology and Population Health, Stanford University School of Medicine,

Stanford, California, USA

${ }^{3}$ Department of Epidemiology and Biostatistics, University of California, San Francisco,

San Francisco, California, USA
Corresponding Author: Alicia R. Riley, PhD, MPH; Department of Sociology, University of California, Santa Cruz, Santa Cruz, California, USA (e-mail: alicia.riley@ucsc.edu).

Funding M. V. K. is supported by the National Institute on Drug Abuse of the National Institutes of Health under Award Number K99DA051534. K. B. D. is supported by intramural funding.

Data Availability Reproducible code is available in an online repository at https://github.com/mkiang/ca_vaccination_impact. When possible, we share aggregated, deidentified data in the same repository; however, our data use agreement prevents us from sharing observations with few deaths.

Declarations:

Conflict of Interest: The authors declare that they do not have a conflict of interest.

\section{REFERENCES}

1. Mackey K. et al. Racial and Ethnic Disparities in COVID-19-Related Infections, Hospitalizations, and Deaths. Ann Intern Med 2020;174:362373.

2. Hughes MM, Wang A, Grossman M, Pun E, Whiteman A.County-Level COVID-19 Vaccination Coverage and Social Vulnerability - United States, December 14, 2020-March 1, 2021. MMWR Morb Mortal Wkly Rep 2021;70:431-436.

3. Neilson $\mathbf{S}$. How the delta surge changed the death divide between California's Republican and Democratic areas. San Francisco Chronicle. https://www.sfchronicle.com/health/article/Are-politicsinfluencing-COVID-19-death-rates-16447486.php. Accessed 9 Sept 2021.

4. CA State Government. Vaccination Progress Data. https://covid19.ca.gov/ vaccination-progress-data/ (Accessed Dec 5, 2021).

5. Bruckner TA. et al. Estimated seroprevalence of SARS-CoV-2 antibodies among adults in Orange County, California. Sci Rep 2021;11:3081.

6. Lopez-Villafana A. Knocking on doors, standing near grocery stores: Groups take grassroots approach to vaccinate Latinos. San Diego UnionTribune 2021

Publisher's Note: Springer Nature remains neutral with regard to jurisdictional claims in published maps and institutional affiliations. 